\title{
Collisionless Relaxation of a Disequilibrated Current Sheet and Implications for Bifurcated Structures
}

\section{Young Dae Yoon ( $\nabla$ yyoon11@postech.ac.kr)}

Pohang Accelerator Laboratory https://orcid.org/0000-0001-8394-2076

Gunsu Yun

Pohang University of Science and Technology (POSTECH) https://orcid.org/0000-0002-1880-5865

James Burch

Southwest Research Institute https://orcid.org/0000-0003-0452-8403

Deirdre Wendel

NASA Goddard Space Flight Center https://orcid.org/0000-0002-1925-9413

\section{Article}

Keywords: Magnetic Phenomena, Phase-space Distributions, Single-particle Orbit Classes, Particle-in-cell Simulations, Bifurcated Current Sheets

Posted Date: March 25th, 2021

DOl: https://doi.org/10.21203/rs.3.rs-343769/v1

License: (1) (1) This work is licensed under a Creative Commons Attribution 4.0 International License. Read Full License

Version of Record: A version of this preprint was published at Nature Communications on June 18th, 2021. See the published version at https://doi.org/10.1038/s41467-021-24006-x. 
1

\title{
Collisionless Relaxation of a Disequilibrated Current Sheet and Implications for Bifurcated Structures
}

\author{
Young Dae Yoon* \\ Pohang Accelerator Laboratory, POSTECH, Pohang, Republic of Korea \\ Gunsu S. Yun* \\ Department of Physics, POSTECH, Pohang, Republic of Korea \\ Deirdre E. Wendel \\ NASA Goddard Space Flight Center, Greenbelt, MD, USA \\ James L. Burch \\ Southwest Research Institute, San Antonio, TX, USA
}

\begin{abstract}
Current sheets are ubiquitous plasma structures that play the crucial role of being energy sources for various magnetic phenomena. Although a plethora of current sheet equilibrium solutions have been found, the collisionless process through which a disequilibrated current sheet relaxes or equilibrates remains largely unknown. Here we show, through analyses of phase-space distributions of single-particle orbit classes and particle-in-cell simulations, that collisionless transitions among the orbit classes are responsible for this process. Bifurcated current sheets, which are readily observed in geospace but whose origins remain controversial, are shown to naturally arise from the equilibration process and thus are likely to be the underlying structures in various phenomena; comparisons of spacecraft observations to particle-in-cell simulations support this fact. The bearing of this result on previous explanations of bifurcated structures is also discussed.
\end{abstract}




\section{I. INTRODUCTION}

12 Current sheets are structures generated by opposing magnetic fields and are ubiquitous 13 in magnetized plasmas such as solar flares[1], the solar wind[2], the heliosphere[3, 4], and ${ }_{14}$ planetary magnetospheres[5, 6]. It is also deeply related to magnetic reconnection[7, 8], a 15 process in which the energy stored in magnetic fields is converted to particle kinetic energies. ${ }_{16}$ Current sheets have thus been subject to extensive research, and a plethora of equilibrium 17 solutions have been found both analytically[9-16] and numerically[17-20].

18 However, there remains an important outstanding question regarding current sheet equi19 libria. Although various equilibrium solutions have been found, the collisionless process 20 through which a disequilibrated current sheet equilibrates remains largely unknown. Such 21 knowledge is crucial because plasmas in general do not start from equilibria, and also because 22 it elucidates how a given system "wants" to evolve in time, even if it does not eventually ${ }_{23}$ equilibrate. In addition, the equilibria that have been found are specific solutions; a com${ }_{24}$ prehensive understanding of the equilibration or relaxation process is necessary in order to ${ }_{25}$ place current sheets in a general context.

26 A commonly observed form of current sheets is a bifurcated current sheet, which has ${ }_{27}$ two current density peaks on either side of the symmetry plane. These were first observed 28 in the Earth's magnetotail by Cluster spacecraft measurements[21, 22] and were initially 29 deemed atypical. Later analyses, however, showed that bifurcated current sheets are actually so extremely common, and that they were detected $\sim 25 \%$ of the time Cluster was in the 31 magnetotail current sheet[23, 24]. Since then, various explanations have been put forth, 32 including flapping motion[25], magnetic reconnection[26, 27], temperature anisotropy[28, 29], 33 Speiser motion[30], and non-adiabatic scattering of particles in a strongly curved magnetic

${ }_{34}$ field[16]. However, there is no consensus on the origin of bifurcated current sheets, which 35 largely remains a mystery despite being readily observed even to this day[31-33].

36 In this paper, the collisionless relaxation process of an initially disequilibrated current 37 sheet is studied. The process is shown in three steps. First, particle orbits in a magnetic з8 field reversal are comprehensively categorized into four orbit classes. Second, the phase39 space distribution of each orbit class and the role each class plays with respect to current 40 sheet density, temperature, and strength are examined. Finally, with the aid of particle41 in-cell simulations, it is shown that transitions among the orbit classes are responsible for 
42 collisionless current sheet relaxation. The final equilibrium is most naturally understood in 43 terms of the relative population of the phase-space distributions of the four orbit classes, 44 instead of closed-form functions such as a Maxwellian.

45 The bearing of this process on the origin of bifurcated current sheets is then discussed. ${ }_{46}$ Two of the orbit classes necessarily exhibit spatially bifurcated structures, and so such ${ }_{47}$ structures naturally arise as a current sheet evolves towards equilibrium via orbit class 48 transitions. An exemplary equilibrium from particle-in-cell simulations is compared with 49 Magnetospheric Multiscale (MMS) measurements of an electron-scale current sheet, and 50 their profiles are shown to agree well. The relevance of the relaxation process to previous 51 explanations of bifurcated current sheets is also discussed.

\section{II. RESULTS}

53 Particle Orbit Classes Let us first examine single-particle dynamics in the renowned ${ }_{54}$ Harris current sheet[9], which is chosen as the system of scrutiny in the present study. It is ${ }_{55}$ described by the following magnetic field profile and distribution function $f_{\sigma}$ for species $\sigma$ ${ }_{56}$ ( $i$ for ions and $e$ for electrons):

$$
\begin{aligned}
\mathbf{B}(x) & =\hat{y} B_{0} \tanh \frac{x}{\lambda}, \\
f_{\sigma}(x, \mathbf{v}, t) & =\left(\frac{1}{2 \pi v_{T \sigma}^{2}}\right)^{3 / 2} \frac{n_{0}}{\cosh ^{2}(x / \lambda)} \\
& \times \exp \left[-\frac{1}{2 v_{T \sigma}^{2}}\left(v_{x}^{2}+v_{y}^{2}+\left(v_{z}-V_{\sigma}\right)^{2}\right)\right],
\end{aligned}
$$

${ }_{57}$ where $B_{0}$ is the asymptotic value of the magnetic field, $\lambda$ is the sheath thickness, $n_{0}$ is the ${ }_{58}$ sheath peak density, and $v_{T \sigma}=\sqrt{k_{B} T_{\sigma} / m_{\sigma}}$ is the species thermal velocity where $T_{\sigma}$ and ${ }_{59} m_{\sigma}$ are respectively the species temperature and mass. $V_{\sigma}$ is the species' mean velocity 60 in the $z$-direction, i.e., its drift velocity. It is also assumed that $T_{i}=T_{e}:=T$ and $V_{i}=$ ${ }_{61}-V_{e}:=V$; the latter can always be made true by choosing a frame of reference where the ${ }_{62}$ electrostatic potential $\phi=0$. Two conditions must be true in order for this system to be 63 an exact solution of the stationary Vlasov equation: (i) $B_{0}=2 \sqrt{\mu_{0} n_{0} k_{B} T}$, which describes ${ }_{64}$ the balance between the peak magnetic pressure $B_{0}^{2} / 2 \mu_{0}$ and the peak thermal pressure ${ }_{65} n_{0} k_{B}\left(T_{i}+T_{e}\right)=2 n_{0} k_{B} T$, and (ii) $\lambda=\lambda_{D} c / V$ where $\lambda_{D}=\sqrt{\epsilon_{0} k_{B} T / n_{0} e^{2}}$ is the Debye 
${ }_{66}$ length and $c$ is speed of light, which determines the equilibrium sheath thickness.

67 The vector potential is chosen to be $\mathbf{A}=-\hat{z} \lambda B_{0} \ln \cosh x / \lambda$. Normalizing length by ${ }_{68} \lambda$, mass by the species mass $m_{\sigma}$, and time by $\omega_{c \sigma}=q_{\sigma} B_{0} / m_{\sigma}$ where $q_{\sigma}$ is the species ${ }_{69}$ charge, then a particle obeys Lagrangian dynamics with the normalized Lagrangian $\bar{L}=$ $70\left(\bar{v}_{x}^{2}+\bar{v}_{y}^{2}+\bar{v}_{z}^{2}\right) / 2-\bar{v}_{z} \ln \cosh \bar{x}$, where barred quantities are normalized to their respective ${ }_{71}$ reference units, i.e., $\bar{L}=L / m_{\sigma} \lambda^{2} \omega_{c \sigma}^{2}, \bar{v}_{x}=v_{x} / \lambda \omega_{c \sigma}$, and $\bar{x}=x / \lambda$. Because $y$ and $z$ are 72 ignorable coordinates, there are three constants of motion, namely the canonical momenta ${ }_{73} \bar{p}_{y}=\partial \bar{L} / \partial \bar{v}_{y}=\bar{v}_{y}$ and $\bar{p}_{z}=\partial \bar{L} / \partial \bar{v}_{z}=\bar{v}_{z}-\ln \cosh \bar{x}$, and the total energy of the parti74 cle $($ recall that $\phi=0), \bar{H}=\left(\bar{v}_{x}^{2}+\bar{v}_{y}^{2}+\bar{v}_{z}^{2}\right) / 2=\bar{v}_{x}^{2} / 2+\left[\bar{p}_{y}^{2}+\left(\bar{p}_{z}+\ln \cosh \bar{x}\right)^{2}\right] / 2$. The 75 normalized effective potential $\chi(\bar{x})$ of the motion in the $x$-direction is therefore $\chi(\bar{x})=$ $76\left[\bar{p}_{y}^{2}+\left(\bar{p}_{z}+\ln \cosh \bar{x}\right)^{2}\right] / 2$.

77 Analyzing the extrema of $\chi(\bar{x})$ shows that it exhibits two shapes depending on the sign of ${ }_{78} \bar{p}_{z}$ : (i) a single-well if $\bar{p}_{z}>0$ (e.g., black line in Fig. 1d), and (ii) a double-well with a local hill 79 at $\bar{x}=0$ if $\bar{p}_{z}<0$ (e.g., black line in Fig. 1a). In case (ii), if a particle does not have enough so energy to overcome the local hill, i.e., $\bar{H}<\chi(0)$ or equivalently $\sqrt{\bar{v}_{x}^{2}+\bar{v}_{z}^{2}}=\bar{v}_{\perp}<-\bar{p}_{z}$, ${ }_{81}$ it oscillates within one of the two wells and does not cross $\bar{x}=0$. In the opposite case 82 where $\bar{v}_{\perp}>-\bar{p}_{z}$, the particle has enough energy to overcome the hill and thus undergoes 8з a full double-well orbit while crossing $\bar{x}=0$. This double-well orbit class can be further ${ }_{84}$ divided into two sub-classes depending on the particle's bounce-period-averaged velocity in ${ }_{85}$ the $z$-direction $\left\langle\bar{v}_{z}\right\rangle$. Because $\left\langle\bar{v}_{z}\right\rangle=\left\langle\bar{p}_{z}\right\rangle+\langle\ln \cosh \bar{x}\rangle$ while $\bar{p}_{z}<0$ is a constant in the case ${ }_{86}$ of a double-well $\chi$, a particle can have either a positive or negative $\left\langle\bar{v}_{z}\right\rangle$ depending on its 87 oscillation amplitude in the $x$-direction; particles with higher energies have higher values of ${ }_{88}\langle\ln \cosh \bar{x}\rangle$ and thus can have positive values of $\left\langle\bar{v}_{z}\right\rangle$.

89 Figure 1 summarizes the four classes of particle orbits. The black lines in Figs. 1a-d show 9o the effective potential $\chi$ of each class, and the three dashed lines in each panel represent the ${ }_{91}$ energies of three particles with differing values of initial $\bar{v}_{x}$ and thus of $\bar{H}$. Each particle is ${ }_{92}$ distinguished by its respective color (blue, red, or cyan). The three lines in Figs. 1e-h show ${ }_{93}$ the motion of the three particles in the left panels in the $x-z$ plane, and the black dots 94 represent their starting positions.

95 Figure 1e represents the non-crossing orbit class[34], hereafter denoted NC, where the ${ }_{96}$ particles are simply $\nabla B$ drifting with $\left\langle\bar{v}_{z}\right\rangle<0$. Figure $1 \mathrm{f}$ represents the class where particles ${ }_{97}$ undergo full double-well motion with $\left\langle\bar{v}_{z}\right\rangle<0$, hereafter denoted DW-. The blue particle in 

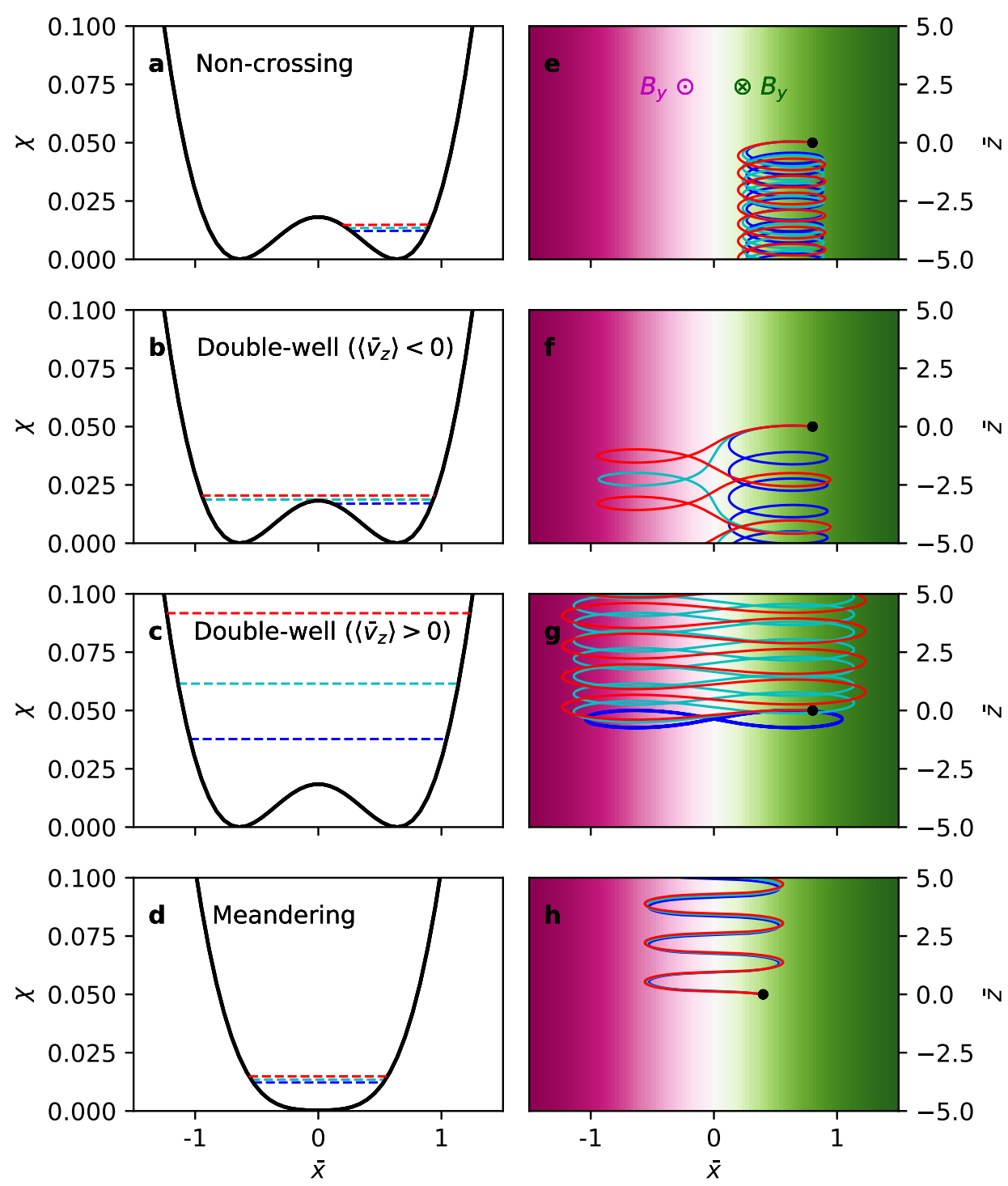

FIG. 1. Four classes of particle orbits and their effective potentials. Effective potentials $\chi$ of the a non-crossing (NC) orbit class, $\mathbf{b}$ double-well orbit class with a negative time-averaged velocity $\left(\left\langle\bar{v}_{z}\right\rangle<0\right.$; DW- $)$, c double-well orbit class with a positive time-averaged velocity $\left(\left\langle\bar{v}_{z}\right\rangle>0\right.$; DW + ), and $\mathbf{d}$ meandering (M) orbit class. e-h Particle orbits in the $\bar{x}-\bar{z}$ plane respectively belong to the four classes in a-d. Three particles are plotted for each class and are labelled by the blue, cyan, and red colors. Each particle's energy is represented by its corresponding color in a-d. The blue particles in $\mathbf{f}$ and $\mathbf{g}$ respectively belong to $\mathrm{NC}$ and $\mathrm{DW}-$ but are plotted to show the $\mathrm{NC} \rightarrow$ $\mathrm{DW}-$ and $\mathrm{DW}-\rightarrow \mathrm{DW}+$ transitions. 
${ }_{98}$ Figure 1f belongs to $\mathrm{NC}$ but is plotted to show the transition from NC to DW-. Figure $1 \mathrm{~g}$ 99 represents the other class where $\left\langle\bar{v}_{z}\right\rangle>0$, hereafter denoted DW+. Again, the blue particle 100 belongs to the DW - class but is plotted to show the transition from DW- to DW+. Figure 101 1h represents the meandering or Speiser orbit class[35] with $\left\langle\bar{v}_{z}\right\rangle>0$, hereafter denoted M. 102 The DW+ class was previously identified in a context with curved magnetic fields as ${ }_{103}$ "cucumber orbits[36, 37]" due to its cucumber shape. Here we have re-identified the class 104 to clarify the physical origin of such motion and to distinguish more clearly between DW+ 105 and DW-, the latter of which does not exhibit cucumber shapes.

106 Phase-Space Distributions Now let us examine how each orbit class is represented 107 in phase space. $10^{8}$ particles were randomly sampled from Eq. 2 with $\bar{V}_{\sigma}=0.005$ and ${ }_{108} \bar{v}_{T \sigma}=0.05$ - these specific values satisfy the equilibrium condition for the Harris sheet. 109 Figure 2 shows the phase space distributions (a-c) and velocity space histograms (d-f) in 110 each velocity direction, and Fig. $2 \mathrm{~g}$ shows the spatial histograms. The orbit classes are 111 distinguished by the black, red, green, and blue colors. The dotted lines and the arrows 112 in the right panels correspond to the mean velocity and the velocity spread (two standard 113 deviations) of each orbit class.

${ }_{114}$ The phase-space distribution of each orbit class has its own contribution to current sheet 115 density, temperature, and strength. The spatial distribution in Fig. $2 \mathrm{~g}$ is related to the 116 density, and the spreads and means of the velocity distributions in Figs. 2d-f are respectively 117 related to the temperature and current strength of each orbit class.

${ }_{118}$ Figure $2 \mathrm{~d}$ shows that the velocity spread and hence the temperature in the $x$-direction, ${ }_{119} T_{x x}$, has the following hierarchy: $\mathrm{NC}<\mathrm{DW}-<\mathrm{DW}+$. This is because the transition from ${ }_{120} \mathrm{NC}$ to DW necessarily involves a passage through the unstable equilibrium as in Fig. 1b, ${ }_{121}$ which in turn involves a breakdown of adiabatic invariance and phase-mixing[38]. $T_{x x}$ of the $122 \mathrm{M}$ class is equal to the overall equilibrium temperature. The mean velocity in the $x$-direction 123 is befittingly zero for all classes due to symmetry.

${ }_{124}$ Figure 2e shows that all classes have the same temperatures and zero mean velocities in 125 the $y$-direction, since $\bar{v}_{y}$ is a constant of motion.

${ }_{126}$ Figure $2 \mathrm{f}$ shows that the temperature in the $z$-direction has the hierarchy $\mathrm{M}<\mathrm{DW}+<$ ${ }_{127} \mathrm{NC}<\mathrm{DW}-$. The $\mathrm{NC}$ and $\mathrm{DW}-$ classes have negative mean velocities and the other two 128 have positive mean velocities.

${ }_{129}$ Figure $2 \mathrm{~g}$ shows that the three non-NC classes are spatially concentrated near the center. 

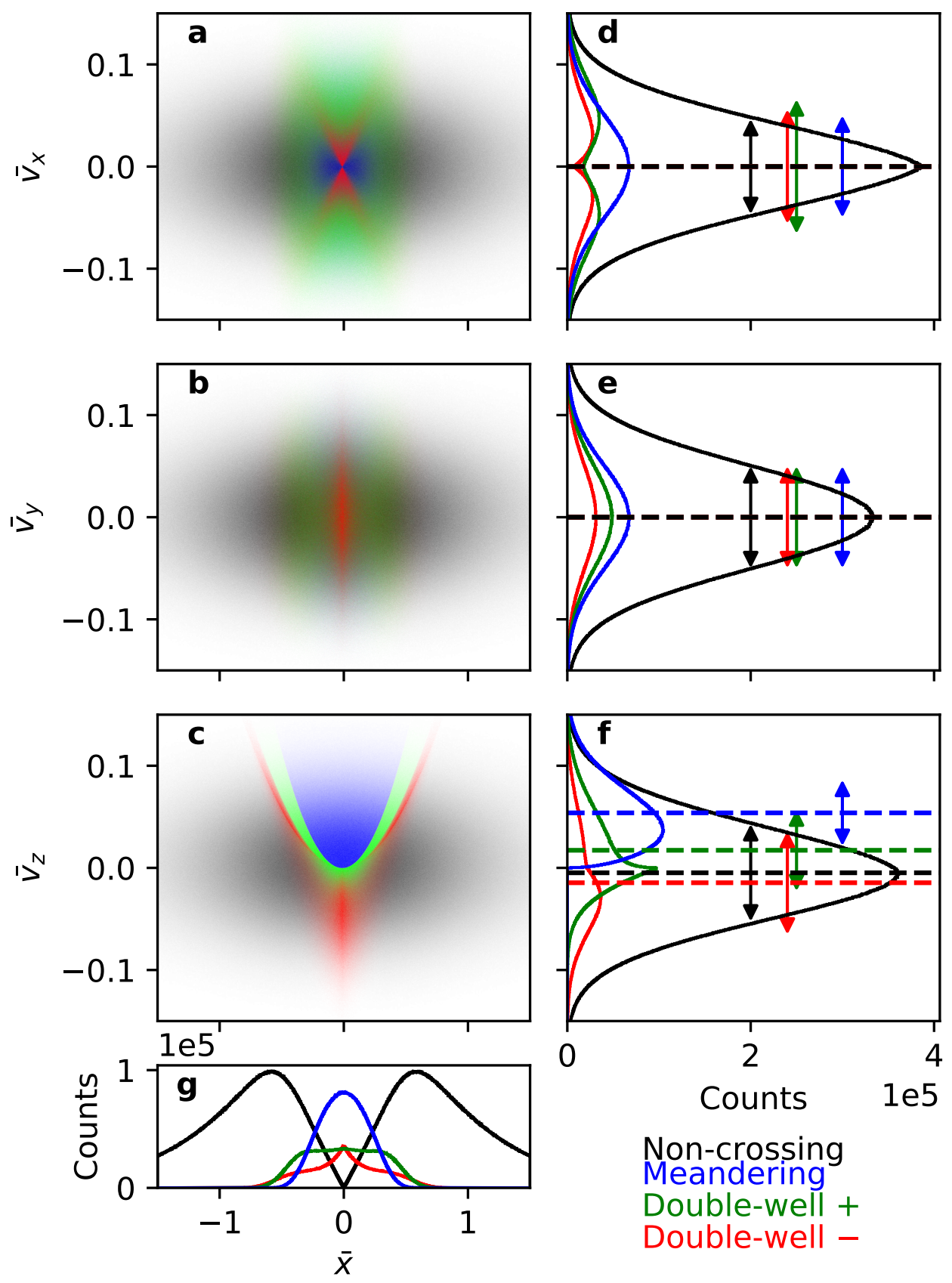

FIG. 2. Particle distribution in phase space, velocity space, and physical space. Phase space distributions of the four orbit classes distinguished by the blue, green, red, and black colors in $\mathbf{a} \bar{x}-\bar{v}_{x}$ space, $\mathbf{b} \bar{x}-\bar{v}_{y}$ space, and $\mathbf{c} \bar{x}-\bar{v}_{z}$ space. Particle histograms in $\mathbf{d} \bar{v}_{x}$, e $\bar{v}_{y}$, and $\mathbf{f} \bar{v}_{z}$. The dotted lines and arrows are respectively the average velocities and two standard deviations of each distribution. g Particle histogram in $\bar{x}$. 
${ }_{130}$ Also, the DW classes have relatively flat-top density profiles compared to the M class, a 131 trait which will be revisited later.

132 Equilibration Process We now have all the ingredients to understand how an initially 133 disequilibrated current sheet equilibrates. Let us consider an under-heated Harris current 134 sheet with a temperature lower than its equilibrium value. In this case, because the thermal 135 pressure at the center is lower than the magnetic pressure at the outskirts, one expects 136 heating and pinching (increase of current density) of the current sheet that lead to a new 137 equilibrium.

${ }_{138}$ Let us first predict how the heating and pinching will happen. Inserting Eqs. 1 and 2 in 139 the Vlasov equation yields

$$
\frac{\partial \ln f_{\sigma}}{\partial \bar{t}}=-2\left(\frac{\bar{V}_{\sigma}}{2 \bar{v}_{T \sigma}^{2}}-1\right) \bar{v}_{x} \tanh \bar{x} .
$$

${ }_{140}$ At equilibrium, the Harris sheet has $\bar{V}_{\sigma}=2 \bar{v}_{T \sigma}^{2}$, which can be confirmed by matching the ${ }_{141}$ current density obtained by $\mathbf{J}=\nabla \times \mathbf{B} / \mu_{0}$ and that obtained by $\mathbf{J}=\sum_{\sigma} q_{\sigma} \int \mathbf{v} f_{\sigma} d^{3} \mathbf{v}$. ${ }_{142}$ However, if the sheet is under-heated so that $2 \bar{v}_{T \sigma}^{2}<\bar{V}_{\sigma}$, then the quantity $\xi:=\bar{V}_{\sigma}-2 \bar{v}_{T \sigma}^{2}$ 143 is positive and Eq. 3 yields a solution linear in a small time interval $\Delta \bar{t}$ :

$$
f_{\sigma} \propto \exp \left[-\frac{1}{2 \bar{v}_{T \sigma}^{2}}\left(\bar{v}_{x}+\xi \Delta \bar{t} \tanh \bar{x}\right)^{2}\right] .
$$

${ }_{144}$ The mean velocity in the $x$-direction is thus $\bar{V}_{x}(\bar{x})=-\xi \Delta \bar{t} \tanh \bar{x}$. At positive $\bar{x}$, particles 145 gain negative $\bar{v}_{x}$ and vice-versa; therefore, the initial linear response of an under-heated ${ }_{146}$ Harris sheet is to bring particles closer to the center by increasing their $\left|\bar{v}_{x}\right|$.

${ }_{147}$ This response induces transitions among particle classes. It is apparent from Fig. 1a-c ${ }_{148}$ that an increase in $\left|\bar{v}_{x}\right|$ moves NC particles to DW- and DW- particles to DW + . Applying 149 the analysis of the phase-space distributions of the four classes in Fig. 2, these class tran150 sitions explain (i) current sheet heating in the $x$-direction, and (ii) current sheet pinching 151 due to increases in both density and mean velocity at the center (note that the velocity 152 decrease from the $\mathrm{NC} \rightarrow \mathrm{DW}$ - transition is more than compensated for in the DW $-\rightarrow$ ${ }_{153} \mathrm{DW}+$ transition). Also, there is no transition to or from the M class because the shape of ${ }_{154} \chi$ is such that a change in $\bar{v}_{x}$ does not induce orbit class transitions.

155 The above analysis only considers linear dynamics assuming that the current sheet profile 156 remains stationary. It is therefore not valid in the nonlinear regime where the profile self157 consistently changes along with orbit class transitions. However, we may infer from the 

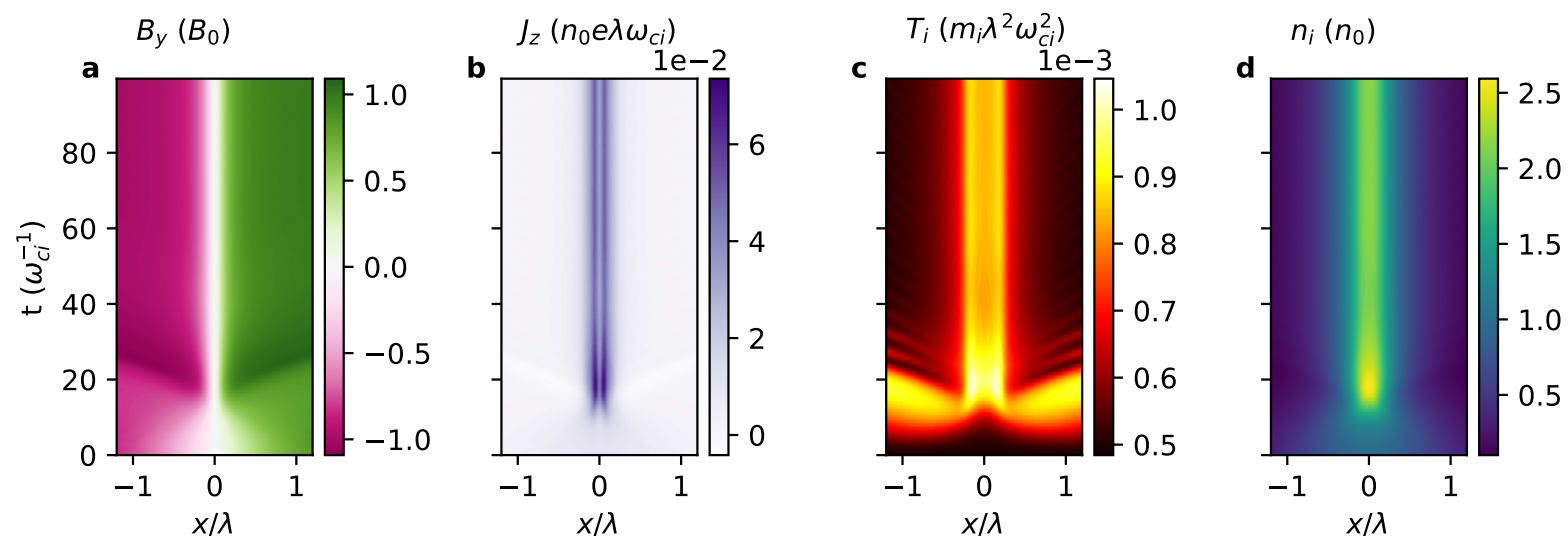

FIG. 3. Streak plots of variables from the particle-in-cell simulation. Streak plots of a the sheared magnetic field $B_{y}$, $\mathbf{b}$ the current density $J_{z}$, $\mathbf{c}$ the ion temperature $T_{i}$, and $\mathbf{d}$ the ion density $n_{i}$ from $t=0$ to $1000 \omega_{c i}^{-1}$.

158 analysis the primary mechanism - at least in the linear regime - underlying current sheet 159 heating and pinching: transitions from the NC class to the DW classes and no transitions 160 to or from the M class.

161 These predictions will now be verified with a one-dimensional particle-in-cell simulation. 162 The initial condition was an under-heated Harris current sheet with a temperature $T=$ ${ }_{163} 0.2 T_{\text {eq }}$ where $T_{\text {eq }}=B_{0}^{2} /\left(4 \mu_{0} n_{0} k_{B}\right)$ is the Harris equilibrium temperature. The initial sheet ${ }_{164}$ thickness was $\lambda=10 d_{i}$ where $d_{i}$ is the collisionless ion skin depth. Figure 3 shows streak 165 plots of $B_{y}, J_{z}$, the ion temperature $T_{i}$, and the ion density $n_{i}$. The current sheet pinches 166 and heats up until $\sim 30 \omega_{c i}^{-1}$, after which it remains steady and thus reaches equilibrium.

${ }_{167}$ Figures $4 \mathrm{a}-\mathrm{c}$ show $f_{i}$ in $x-v_{x}$ space at $t=0,10,100 \omega_{c i}^{-1}$, respectively. Figure $4 \mathrm{~b}$ confirms 168 the initial response of the under-heated current sheet as predicted by Eq. 4, namely the 169 focussing of the particles towards the center. Figure 4c shows the equilibrium reached by $170 f_{i}$, and Fig. $4 \mathrm{~d}$ shows the difference $\left(\Delta f_{i}\right)$ between the initial state (Fig. 4a) and the 171 equilibrium state (Fig. 4c). Comparing Fig. 4d to Fig. 2a, it is apparent that the NC 172 class de-populates and migrates to the DW classes. The dynamics in the simulation is fully 173 nonlinear, so transition to the M class also occurs, albeit less significantly than the main ${ }_{174} \mathrm{NC} \rightarrow$ DW transition.

175 Figures $4 \mathrm{e}-\mathrm{g}$ and $4 \mathrm{i}$ are the same as Figs. 4a-d except that they are in $x-v_{z}$ space. 176 Again, the $\mathrm{NC} \rightarrow \mathrm{DW}$ transition is evident from a comparison to the pronounced Y-shape 

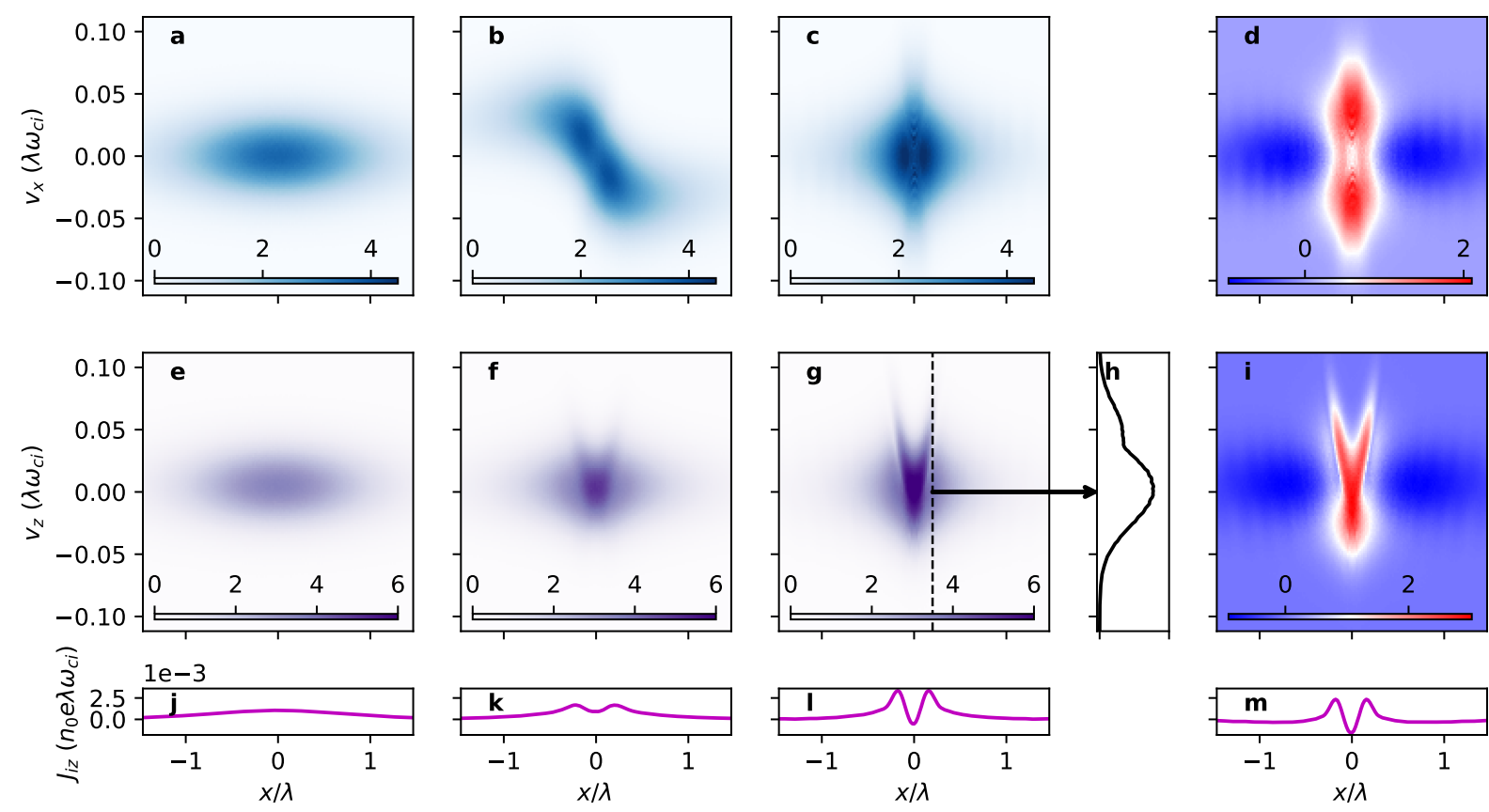

FIG. 4. Time evolution of the ion distribution function from the particle-in-cell simulation. Ion distribution function $f_{i}$ in $x-v_{x}$ space at $\mathbf{a} t=0, \mathbf{b} t=10 \omega_{c i}^{-1}$, and $\mathbf{c} t=100 \omega_{c i}^{-1}$. $\mathbf{d}$ The difference $\left(\Delta f_{i}\right)$ between $f_{i}$ in $\mathbf{c}$ and $\mathbf{a}$. e-g and $\mathbf{i}$ are respectively the same as a-c and $\mathbf{d}$, except in $x-v_{z}$ space. $\mathbf{h}$ A slice through the dotted line in $\mathbf{g}$. $\mathbf{j}-\mathbf{l}$ The ion current density $J_{i z}$ obtained by taking the first velocity moment of $\mathbf{e}-\mathbf{g}$. $\mathbf{m}$ The difference between $\mathbf{j}$ and $\mathbf{l}$.

177 of the phase-space distribution of the DW classes (Fig. 2c). Therefore, we have confirmed 178 that collisionless equilibration of an under-heated Harris current sheet is mainly due to orbit 179 class transitions from NC to DW.

180 It is clear from Figs. 4c and $4 \mathrm{~g}$ that the final equilibrium is most naturally described by the 181 relative population in each orbit class, rather than, e.g., Maxwellian or kappa distributions. 182 Figure $4 \mathrm{~h}$ shows the distribution in $v_{z}$ at $x=0.2 \lambda$, whose profile is clearly non-Maxwellian. ${ }_{183}$ Note that electrons also mainly transition from NC to DW in this process because the ${ }_{184}$ orbit classes apply generally for any species. Figure 4 is therefore qualitatively applicable 185 to electrons, except that their velocities change signs due to their negative charge.

${ }_{186}$ Origin of Bifurcated Current Sheets Figures $4 \mathrm{j}-\mathrm{l}$ show the time evolution of the 187 ion current density in the $z$-direction, $J_{i z}$, and Fig. $4 \mathrm{~m}$ shows the difference between the 188 initial and final $J_{i z}$. The bifurcated structure is evident, which naturally arises from the 189 pronounced Y-shape of the phase-space distribution of the DW classes to which particles 
190 migrate from the NC class. The total current density - mainly carried by the electrons191 is also bifurcated, as shown in Fig. 3b. Bifurcated current structures are thus natural 192 by-products of the collisionless equilibration process of a current sheet.

193 Let us compare the simulation results with a current sheet detected by the Magnetospheric 194 Multiscale (MMS) mission[39] from 20:24:00 to 20:24:15 UT on 17 June 2017, when the space195 craft was located at $(-19.4,-10.4,5.5) R_{e}$ in Geocentric Solar Ecliptic (GSE) coordinates 196 while crossing the magnetotail plasma sheet from the southern to the northern hemisphere. ${ }_{197}$ This sheet has also been examined in previous studies under different contexts[33, 40, 41]. ${ }_{198}$ The focus will now be on electrons instead of ions because the observed current sheet has 199 an electron scale thickness $\left(<10 d_{e}\right)$, and as a confirmation that electrons have similar orbit 200 class transition dynamics to that of ions.

201 Figure 5 shows a side-by-side comparison of the current sheet detected by MMS and that 202 from the particle-in-cell simulations. The data are presented using a local coordinate system, ${ }_{203} L M N$. The sheared magnetic field is in the $L$-direction, and $M$ and $N$ are respectively 204 parallel and normal to the current sheet. The current is carried mainly by the electrons in 205 both the observation and the simulation. The finite electron outflow $v_{e L}$ in Fig. 5b indicates 206 that the observed current sheet is undergoing magnetic reconnection, whereas the simulated 207 current sheet, being one-dimensional, is not. Reconnection induces perpendicular electron 208 heating at the sheet center[42], which explains the central increase of $T_{e M M}$ in Fig. 5d 209 relative to Fig. 5j. The relative increase of $T_{e L L}$ at the outskirts in Fig. $5 \mathrm{~d}$ is also attributed 210 to reconnection-induced parallel electron heating[42].

${ }_{211}$ Apart from such reconnection-related dissimilarities, the observed and simulated profiles 212 agree strikingly well, including the bifurcated current structure. In particular, the simulated 213 equilibrium explains the central dip and increased outskirts of the electron temperature 214 tensor element $T_{e M M}$ relative to $T_{e N N}$, as shown in Figs. $5 \mathrm{~d}$ and $5 \mathrm{j}$. The profile of $T_{e N N}-$ ${ }_{215} T_{e M M}$ in Fig. 5e is remarkably reproduced by Fig. 5k, except for the relative central dip 216 in Fig. 5e due to the reconnection-induced increase of $T_{e M M}$. Same goes for the pressure 217 tensor elements $P_{e M M}, P_{e N N}$, and $P_{e N N}-P_{e M M}$ (Figs. 5f and 5l).

${ }_{218}$ The increased amount of electron population in the DW classes is shown not only by the 219 current, temperature, and pressure structures, but also by the density plateau in Fig. 5c 220 and $5 \mathrm{i}$ which is due to migrations to the DW classes (see Fig. $2 \mathrm{~g}$ ). This density plateau was 221 also observed in Cluster measurements[25]. 

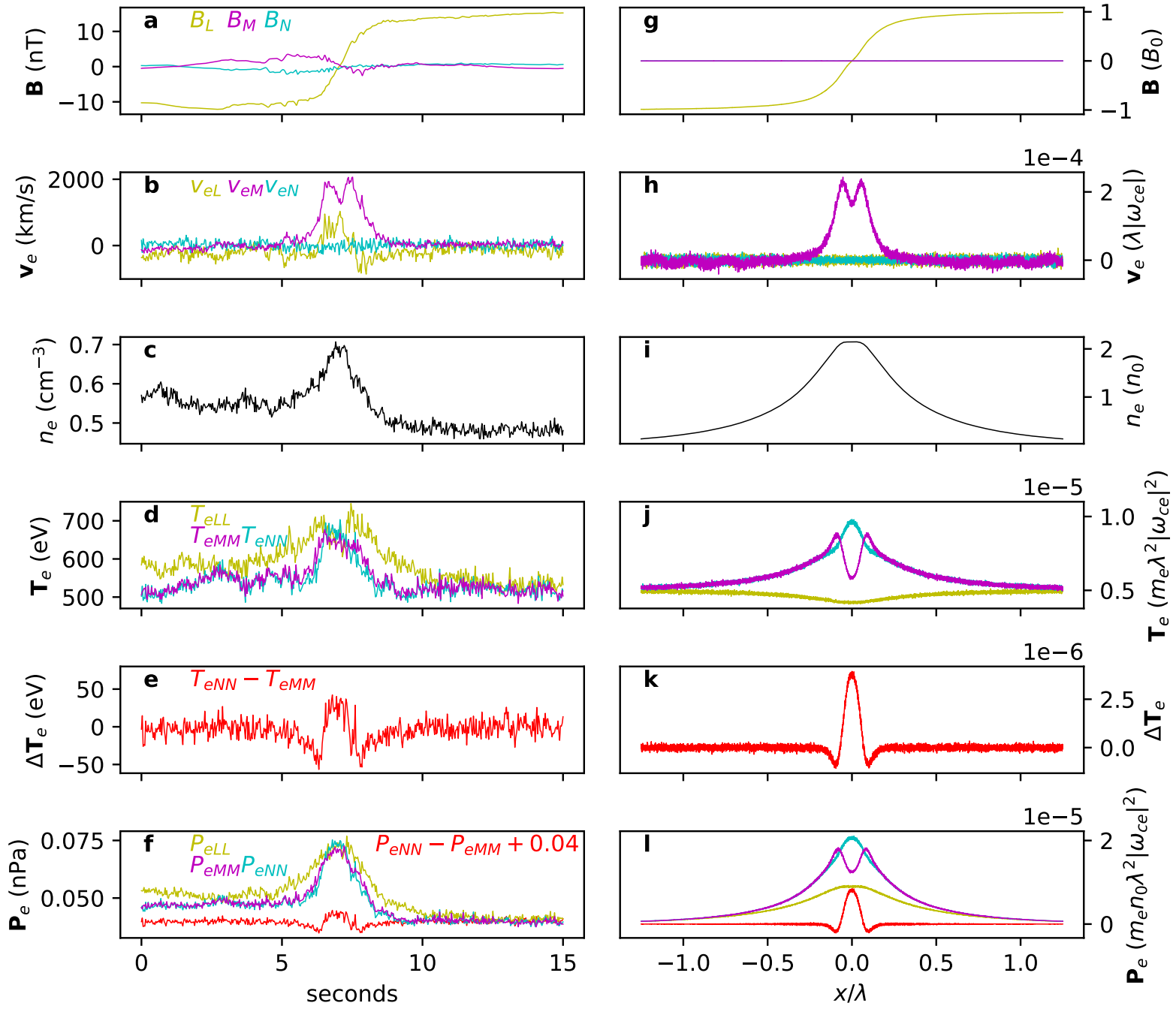

FIG. 5. Comparison of a current sheet detected by MMS to that from the particle-incell simulation. a-f Sequentially, the magnetic field $\mathbf{B}$, electron velocity $\mathbf{v}_{e}$, electron density $n_{e}$, diagonal elements of the electron temperature tensor $\mathbf{T}_{e}$, the difference between the temperature tensor elements $T_{e N N}-T_{e M M}$, and diagonal elements of the electron pressure tensor $\mathbf{P}_{e}$ and $P_{e N N}-$ $P_{e M M}$ (shifted up by 0.04) detected by the Magnetospheric Multiscale spacecraft from 20:24:00 to 20:24:15 UT on 17 June 2017. The $x$-axis is seconds from 20:24:00 UT. g-l Quantities from the particle-in-cell simulation respectively corresponding to a-f.

\section{III. DISCUSSION}

223 Although the new kinetic equilibrium has been presented as an example of bifurcated ${ }_{224}$ current sheets, we are not claiming that all such sheets are in equilibrium states. Instead, 
225 the claim is that bifurcated structures are natural repercussions of the collisionless current 226 sheet equilibration process and so are likely to be observed in a variety of phenomena as 227 the underlying structure. As mentioned in the Introduction, numerous explanations for 228 bifurcated current sheets have been put forth; these explanations will now be unravelled in 229 relation to the relaxation process.

230 Magnetic reconnection has been one of the proposed causes of bifurcated current sheets[26, $\left.{ }_{231} 43\right]$, but many such sheets were also observed without any fast flows[25, 44] which are sig232 natures of reconnection. Nevertheless, a statistical study indicated that the thinner the 233 structures are, the more likely they are to be bifurcated[24]. These observations can be 234 unified by the fact that thinner current sheets are more likely to involve sub-skin-depth 235 collisionless dynamics, which is favorable for the occurrence of both collisionless reconnec${ }_{236}$ tion and the present collisionless relaxation process. A possible scenario is one where an ${ }_{237}$ initially thick, under-heated current sheet equilibrates to a thin, sub-skin-depth bifurcated 238 structure, which then undergoes collisionless reconnection. It fact, the initial condition for 239 reconnection in collisionless situations is more likely to be the equilibrium presented here 240 than widely-used specific solutions such as the Harris sheet[45]. If the sheet does not thin 241 enough for reconnection to occur, then it may remain bifurcated and steady.

${ }_{242}$ Flapping motion was also observed in conjunction with bifurcated current sheets $[25$, ${ }_{243} 27$. This motion involves fast thinning and thickening of the sheet[27]. Such fast motion 244 will naturally induce bifurcation via two possible scenarios: (i) disequilibration of current 245 sheets, followed by relaxation via spontaneous orbit classes transitions, or (ii) unspontaneous 246 transitions driven by the external source that thins the sheet.

$247 \quad$ Equilibria involving anisotropic temperatures have also been shown to exhibit bifurcated 248 structures[13, 28, 29], but the source of the anisotropy was not clear so the amount of 249 anisotropy had been set $a d-h o c$. The present collisionless relaxation process naturally induces 250 temperature anisotropy, which is thus an innate result of the equilibration process rather 251 than a cause of bifurcated structures.

252 Speiser motion (M class) was also attributed to bifurcated structures[17, 30]. However, 253 it is clear from Fig. 2 that the $M$ class cannot contribute to bifurcated structures if the ${ }_{254}$ density is peaked near the center, unless the density itself is bifurcated[17] and/or heavier 255 species are taken into account[30].

${ }_{256}$ Some studies $[16,36,37]$ invoke non-adiabatic scattering of particles from M to DW+ via 
257 a slow diffusive process in current sheet equilibria. However, the diffusion coefficient of such 258 process is zero for $B_{x}=0$ because it scales with the curvature parameter $\kappa$ (cf. equation 7 259 of Zelenyi et al.[37]). We have shown here that neither curved magnetic fields nor diffusive 260 processes are necessary; simply choosing a disequilibrated initial state is sufficient for the 261 development of bifurcated structures, although diffusion due to field curvature may aid the 262 process.

263 In summary, the collisionless relaxation process of a disequilibrated current sheet was 264 studied. The process is most naturally understood by orbit class transitions, which were 265 analytically predicted and numerically verified. The relaxation mechanism was identified as 266 the origin of bifurcated current sheets, and the significance of this identification in regards 267 to previous explanations of bifurcated structures was discussed.

\section{${ }_{268}$ IV. METHODS}

\section{Sampling from and categorization of the Harris distribution function Particle} 270 positions and velocities were sampled from Eq. 2 using the numpy . random package in Python 271 3.8. Particles with $\bar{p}_{z}>0$ and $\bar{p}_{z}<-\bar{v}_{\perp}$ were categorized into $\mathrm{M}$ and NC, respectively. For 272 the rest of the particles that belong to the DW classes, the following steps were taken to 273 further categorize them.

274 First, a simple analysis of the Hamiltonian of each particle shows that its oscillation 275 amplitude in the $x$-direction is given by $\bar{x}_{\max }=\operatorname{arccosh}\left(\exp \left[\bar{v}_{\perp}-\bar{p}_{z}\right]\right)$. The bounce-period276 averaged $\bar{v}_{z}$ of the particle is then given by

$$
\begin{aligned}
\left\langle\bar{v}_{z}\right\rangle & =\frac{2}{T_{0}} \int_{-\bar{x}_{\max }}^{\bar{x}_{\max }} \frac{\bar{v}_{z}}{\bar{v}_{x}} d \bar{x}, \\
& =\frac{2}{T_{0}} \int_{-\bar{x}_{\max }}^{\bar{x}_{\max }} \frac{\bar{p}_{z}+\ln \cosh \bar{x}}{\sqrt{\left(\bar{p}_{z}+\ln \cosh \bar{x}_{\max }\right)^{2}-\left(\bar{p}_{z}+\ln \cosh \bar{x}\right)^{2}}} d \bar{x},
\end{aligned}
$$

277 where $T_{0}=2 \int_{-\bar{x}_{\max }}^{\bar{x}_{\max }} d \bar{x} / \bar{v}_{x}$ is the bounce period. Only the sign of $\left\langle\bar{v}_{z}\right\rangle$ matters here, so the in278 tegral in Eq. 6 was evaluated numerically for each particle using the scipy. integrate.quad 279 package in Python 3.8. Particles with positive $\left\langle\bar{v}_{z}\right\rangle$ were categorized into DW + , and the rest 280 into DW-.

${ }_{281}$ Particle-in-cell simulation The open-source, fully-relativistic particle-in-cell code, 
${ }_{282}$ SMILEI[46], was used. The 1D simulation domain was $10 \lambda=100 d_{i}$ long and was divided ${ }_{283}$ into $2^{15}=32,768$ grid points. Open boundary conditions (Silver-Müller) were employed for 284 the electromagnetic fields in the $x$-direction, and periodic boundary conditions were imposed 285 for the fields in the $y$-direction and for the particles. 10,000 particles were placed per cell 286 per species, so about $6 \times 10^{8}$ particles were simulated with a mass ratio $m_{i} / m_{e}=100$. The 287 simulation run with a frequency ratio of $\omega_{c e} / \omega_{p e}=5$ is shown in this paper for clarity of 288 presentation; ratios as low as $\omega_{c e} / \omega_{p e}=0.2$ were also tried, but lower ratios simply increased 289 the duration of plasma oscillations that either damp or travel away from the center without 290 any noticeable effect on the core relaxation mechanism. The initial conditions were Eqs. 1 291 and 2 , and the electrostatic potential $\phi=0$. The initial temperature was set as one-fifth 292 of the Harris equilibrium temperature, i.e., $T=0.2 T_{\text {eq }}$ where $T_{\text {eq }}=B_{0}^{2} /\left(4 \mu_{0} n_{0} k_{B}\right)$ is the 293 temperature that yields the Harris equilibrium. The simulation time was $t_{\max }=100 \omega_{c i}^{-1}$ 294 with a time step $\Delta t=7.63 \times 10^{-4} \omega_{c i}^{-1}$.

${ }_{295}$ The simulations were run on the KAIROS computer cluster at Korea Institute of Fusion 296 Energy.

297 MMS data and local LMN coordinates Data from MMS2, MMS3, and MMS4 from 298 20:24:00 to 20:24:15 UT on June 17, 2017 were averaged to yield the profiles shown in Figs. 2995 a-e. MMS1 data were omitted because the current density did not exhibit an obvious bi300 furcated structure. The magnetic field data were collected by the Fluxgate Magnetometer 301 instrument[47] and the plasma data by the Fast Plasma Investigation instrument[48]. The 302 local LMN coordinate system is obtained from a minimum variance analysis[49] of the aver303 aged raw data which are in Geocentric Solar Ecliptic (GSE) coordinates. The values of the 304 unit vectors in GSE coordinates are $L=(0.942,0.308,-0.130), M=(0.194,-0.189,0.963)$, 305 and $N=(0.272,-0.932,-0.238)$ in GSE coordinates. $L$ is the direction of the sheared mag306 netic field, $N$ is the direction normal to the current sheet, and $L M N$ form a right-handed 307 coordinate system.

\section{DATA AVAILABILITY}

309 MMS data are publicly available from https://lasp.colorado.edu/mms/sdc/public. The 310 data from the PIC simulations are available from https://doi.org/10.5281/zenodo.4607112. 
SMILEI[46] is an open-source PIC code available from https://smileipic.github.io/Smilei. 313 MMS data were analyzed using the pySPEDAS package, available from https://github.com/spedas/pyspeda 314 The codes used in the data analyses are available from Y.D.Y. upon reasonable request.

[1] Linhui Sui and Gordon D. Holman. Evidence for the Formation of a Large-Scale Current Sheet in a Solar Flare. The Astrophysical Journal, 596(2):L251-L254, oct 2003.

[2] Steven J. Schwartz, Chris P. Chaloner, Peter J. Christiansen, Andrew J. Coates, David S. Hall, Alan D. Johnstone, M. Paul Gough, Andrew J. Norris, Richard P. Rijnbeek, David J. Southwood, and Les J.C. Woolliscroft. An active current sheet in the solar wind. Nature, 318(6043):269-271, nov 1985.

[3] J. Todd Hoeksema, John M. Wilcox, and Philip H. Scherrer. Structure of the Heliospheric Current Sheet: 1978-1982. Journal of Geophysical Research, 88(A12):9910-9918, 1983.

[4] Edward J. Smith. The heliospheric current sheet. Journal of Geophysical Research: Space Physics, 106(A8):15819-15831, aug 2001.

[5] T. W. Speiser. Magnetospheric current sheets. Radio Science, 8(11):973-977, nov 1973.

[6] Krishan K. Khurana and Hannes K. Schwarzl. Global structure of Jupiter's magnetospheric current sheet. Journal of Geophysical Research: Space Physics, 110(A7):A07227, 2005.

[7] Masaaki Yamada, Hantao Ji, Scott Hsu, Troy Carter, Russell Kulsrud, and Fedor Trintchouk. Experimental investigation of the neutral sheet profile during magnetic reconnection. Physics of Plasmas, 7(5):1781-1787, may 2000.

[8] Masaaki Yamada, Russell Kulsrud, and Hantao Ji. Magnetic reconnection. Reviews of Modern Physics, 82(1):603-664, mar 2010.

[9] E. G. Harris. On a plasma sheath separating regions of oppositely directed magnetic field. Il Nuovo Cimento, 23(1):115-121, jan 1962.

[10] J. R. Kan. On the structure of the magnetotail current sheet. Journal of Geophysical Research, 78(19):3773-3781, jul 1973.

37 [11] Paul J. Channell. Exact Vlasov-Maxwell equilibria with sheared magnetic fields. Physics of Fluids, 19(10):1541-1545, 1976. 
339 [12] B. Lembège and R. Pellat. Stability of a thick two-dimensional quasineutral sheet. Physics of $340 \quad$ Fluids, 25(11):1995, 1982.

341 [13] A P Kropotkin, H V Malova, and M I Sitnov. Self-consistent structure of a thin anisotropic

342

343

344 [14] Peter H. Yoon, Anthony T. Y. Lui, and Robert B. Sheldon. On the current sheet model with $345 \quad \kappa$ distribution. Physics of Plasmas, 13(10):102108, oct 2006.

346 [15] Michael G. Harrison and Thomas Neukirch. One-Dimensional Vlasov-Maxwell Equilibrium for 347 the Force-Free Harris Sheet. Physical Review Letters, 102(13):135003, apr 2009.

348 [16] L. M. Zelenyi, H. V. Malova, A. V. Artemyev, V. Yu Popov, and A. A. Petrukovich. Thin

357 [20] Luxiuyuan Jiang and San Lu. Externally driven bifurcation of current sheet: A particle-in-cell

359 [21] A. Runov, R. Nakamura, W. Baumjohann, T. L. Zhang, M. Volwerk, H.-U. Eichelberger, and

367 [24] S. M. Thompson, M. G. Kivelson, M. El-Alaoui, A. Balogh, H. Réme, and L. M. Kistler. current sheets in collisionless plasma: Equilibrium structure, plasma instabilities, and particle acceleration. Plasma Physics Reports, 37(2):118-160, feb 2011. sheet. Planetary and Space Science, 20(10):1555-1568, oct 1972. dimensional tail current sheet. Journal of Geophysical Research, 97(A11):16773, nov 1992. A. Balogh. Cluster observation of a bifurcated current sheet. Geophysical Research Letters, $30(2): 3-6$, jan 2003 .

[22] A. Runov, R. Nakamura, W. Baumjohann, R. A. Treumann, T. L. Zhang, and M. Volwerk. Current sheet structure near magnetic X-line observed by Cluster. Geophysical Research Letters, 30(11):1579, 2003.

[23] Yoshihiro Asano. How typical are atypical current sheets? Geophysical Research Letters, 32(3):L03108, 2005.

Bifurcated current sheets: Statistics from Cluster magnetometer measurements. Journal of Geophysical Research, 111(A3):A03212, 2006. 
370 [25] V. Sergeev, A. Runov, W. Baumjohann, R. Nakamura, T. L. Zhang, M. Volwerk, A. Balogh, 371 H. Rème, J. A. Sauvaud, M. André, and B. Klecker. Current sheet flapping motion and $372 \quad$ structure observed by Cluster. Geophysical Research Letters, 30(6):2-5, mar 2003.

373 [26] M. Hoshino, A. Nishida, T. Mukai, Y. Saito, T. Yamamoto, and S. Kokubun. Structure of 374 plasma sheet in magnetotail: Double-peaked electric current sheet. Journal of Geophysical 375 Research: Space Physics, 101(A11):24775-24786, nov 1996.

376 [27] Y. Asano, T. Mukai, M. Hoshino, Y. Saito, H. Hayakawa, and T. Nagai. Current sheet 377 structure around the near-Earth neutral line observed by Geotail. Journal of Geophysical 378 Research: Space Physics, 109(A2):1-18, feb 2004.

379 [28] M. I. Sitnov, P. N. Guzdar, and M. Swisdak. A model of the bifurcated current sheet. Geo$380 \quad$ physical Research Letters, 30(13):10-13, jul 2003.

381 [29] L. M. Zelenyi, H. V. Malova, V. Yu Popov, D. Delcourt, and A. S. Sharma. Nonlinear equi-

382 librium structure of thin currents sheets: influence of electron pressure anisotropy. Nonlinear Processes in Geophysics, 11(5/6):579-587, nov 2004.

[30] Don E George and Jörg-Micha Jahn. Energized Oxygen in the Magnetotail: Current Sheet Bifurcation From Speiser Motion. Journal of Geophysical Research: Space Physics, 125(2):e2019JA027339, feb 2020.

[31] J. L. Burch and T. D. Phan. Magnetic reconnection at the dayside magnetopause: Advances with MMS. Geophysical Research Letters, 43(16):8327-8338, aug 2016.

[32] M. Zhou, H. Y. Man, Z. H. Zhong, X. H. Deng, Y. Pang, S. Y. Huang, Y. Khotyaintsev, C. T. Russell, and B. Giles. Sub-ion-scale Dynamics of the Ion Diffusion Region in the Magnetotail: MMS Observations. Journal of Geophysical Research: Space Physics, 124(10):7898-7911, oct 2019 .

[33] Rongsheng Wang, Quanming Lu, San Lu, Christopher T. Russell, J. L. Burch, Daniel J. Gershman, W. Gonzalez, and Shui Wang. Physical Implication of Two Types of Reconnection Electron Diffusion Regions With and Without Ion-Coupling in the Magnetotail Current Sheet. Geophysical Research Letters, 47(21):e2020GL088761, nov 2020.

8 Research, 76(34):8211-8222, dec 1971.

[35] T. W. Speiser. Particle trajectories in model current sheets: 1. Analytical solutions. Journal of Geophysical Research, 70(17):4219-4226, sep 1965. 
401 [36] Jörg Büchner and Lev M. Zelenyi. Regular and chaotic charged particle motion in magneto-

405

421 [42] M. A. Shay, C. C. Haggerty, T. D. Phan, J. F. Drake, P. A. Cassak, P. Wu, M. Oieroset, taillike field reversals: 1. Basic theory of trapped motion. Journal of Geophysical Research, 94(A9):11821, 1989.

37] L. M. Zelenyi, H. V. Malova, and V. Yu Popov. Splitting of thin current sheets in the Earth's magnetosphere. Journal of Experimental and Theoretical Physics Letters, 78(5):296-299, sep 2003.

[38] John R Cary, D F Escande, and J L Tennyson. Adiabatic-invariant change due to separatrix crossing. Physical Review A, 34(5):4256-4275, nov 1986.

[39] J. L. Burch, T. E. Moore, R. B. Torbert, and B. L. Giles. Magnetospheric Multiscale Overview and Science Objectives. Space Science Reviews, 199(1-4):5-21, mar 2016.

[40] Rongsheng Wang, Quanming Lu, Rumi Nakamura, Wolfgang Baumjohann, Can Huang, Christopher T. Russell, J. L. Burch, Craig J. Pollock, Dan Gershman, R. E. Ergun, Shui Wang, P. A. Lindqvist, and Barbara Giles. An Electron-Scale Current Sheet Without Bursty Reconnection Signatures Observed in the Near-Earth Tail. Geophysical Research Letters, 45(10):4542-4549, may 2018.

[41] San Lu, Rongsheng Wang, Quanming Lu, V. Angelopoulos, R. Nakamura, A. V. Artemyev, P. L. Pritchett, T. Z. Liu, X.-J. Zhang, W. Baumjohann, W. Gonzalez, A. C. Rager, R. B. Torbert, B. L. Giles, D. J. Gershman, C. T. Russell, R. J. Strangeway, Y. Qi, R. E. Ergun, P.A. Lindqvist, J. L. Burch, and Shui Wang. Magnetotail reconnection onset caused by electron kinetics with a strong external driver. Nature Communications, 11(1):5049, dec 2020.

M. Swisdak, and K. Malakit. Electron heating during magnetic reconnection: A simulation scaling study. Physics of Plasmas, 21(12), 2014.

[43] J. T. Gosling and A. Szabo. Bifurcated current sheets produced by magnetic reconnection in the solar wind. Journal of Geophysical Research: Space Physics, 113(A10):1-8, oct 2008.

[44] Y. Asano, T. Mukai, M. Hoshino, Y. Saito, H. Hayakawa, and T. Nagai. Evolution of the thin current sheet in a substorm observed by Geotail. Journal of Geophysical Research: Space Physics, 108(A5):1-10, may 2003.

[45] J. Birn, J. F. Drake, M. A. Shay, B. N. Rogers, R. E. Denton, M. Hesse, M. Kuznetsova, Z. W. Ma, A. Bhattacharjee, A. Otto, and P. L. Pritchett. Geospace Environmental Modeling (GEM) Magnetic Reconnection Challenge. Journal of Geophysical Research: Space Physics, 
106(A3):3715-3719, mar 2001.

[46] J. Derouillat, A. Beck, F. Pérez, T. Vinci, M. Chiaramello, A. Grassi, M. Flé, G. Bouchard, I. Plotnikov, N. Aunai, J. Dargent, C. Riconda, and M. Grech. SMILEI: A collaborative, opensource, multi-purpose particle-in-cell code for plasma simulation. Computer Physics Communications, 222:351-373, jan 2018.

[47] R. B. Torbert, C. T. Russell, W. Magnes, R. E. Ergun, P.-A. Lindqvist, O. LeContel, H. Vaith, J. Macri, S. Myers, D. Rau, J. Needell, B. King, M. Granoff, M. Chutter, I. Dors, G. Olsson, Y. V. Khotyaintsev, A. Eriksson, C. A. Kletzing, S. Bounds, B. Anderson, W. Baumjohann, M. Steller, K. Bromund, Guan Le, R. Nakamura, R. J. Strangeway, H. K. Leinweber, S. Tucker, J. Westfall, D. Fischer, F. Plaschke, J. Porter, and K. Lappalainen. The FIELDS Instrument Suite on MMS: Scientific Objectives, Measurements, and Data Products. Space Science Reviews, 199(1-4):105-135, mar 2016.

[48] C. Pollock, T. Moore, A. Jacques, J. Burch, U. Gliese, Y. Saito, T. Omoto, L. Avanov, A. Barrie, V. Coffey, J. Dorelli, D. Gershman, B. Giles, T. Rosnack, C. Salo, S. Yokota, M. Adrian, C. Aoustin, C. Auletti, S. Aung, V. Bigio, N. Cao, M. Chandler, D. Chornay, K. Christian, G. Clark, G. Collinson, T. Corris, A. De Los Santos, R. Devlin, T. Diaz, T. Dickerson, C. Dickson, A. Diekmann, F. Diggs, C. Duncan, A. Figueroa-Vinas, C. Firman, M. Freeman, N. Galassi, K. Garcia, G. Goodhart, D. Guererro, J. Hageman, J. Hanley, E. Hemminger, M. Holland, M. Hutchins, T. James, W. Jones, S. Kreisler, J. Kujawski, V. Lavu, J. Lobell, E. LeCompte, A. Lukemire, E. MacDonald, A. Mariano, T. Mukai, K. Narayanan, Q. Nguyan, M. Onizuka, W. Paterson, S. Persyn, B. Piepgrass, F. Cheney, A. Rager, T. Raghuram, A. Ramil, L. Reichenthal, H. Rodriguez, J. Rouzaud, A. Rucker, Y. Saito, M. Samara, J.-A. Sauvaud, D. Schuster, M. Shappirio, K. Shelton, D. Sher, D. Smith, K. Smith, S. Smith, D. Steinfeld, R. Szymkiewicz, K. Tanimoto, J. Taylor, C. Tucker, K. Tull, A. Uhl, J. Vloet, P. Walpole, S. Weidner, D. White, G. Winkert, P.-S. Yeh, and M. Zeuch. Fast Plasma Investigation for Magnetospheric Multiscale. Space Science Reviews, 199(1-4):331-406, mar 2016.

[49] Buo Sonnerup and Maureen Scheible. Minimum and maximum variance analysis. In Analysis methods for multi-spacecraft data, volume 001, pages 185-220. 1998. 
461 This work was supported by the National Research Foundation of Korea under grant 462 nos. NRF-2019R1C1C1003412, NRF-2019R1A2C1004862, and 2019M1A7A1A03088456. ${ }_{463}$ We thank the entire MMS team and MMS Science Data Center for providing the high${ }_{464}$ quality data for this study. We also thank J. M. Kwon, J. H. Kim, and Korea Institute of 465 Fusion Energy for providing the computer resources.

\section{${ }_{466}$ AUTHOR CONTRIBUTIONS}

${ }_{467}$ Y.D.Y. conceived the central idea, performed the simulations and theoretical analysis, 468 analyzed the spacecraft data, and wrote the manuscript based on extensive discussions with ${ }_{469}$ G.S.Y. D.E.W. contributed to the interpretation of the simulation and observation results, 470 as well as to the revision of the draft. J.L.B. oversaw the MMS project and provided general 471 guidance.

472 Correspondence and requests for materials should be addressed to Y.D.Y or G.S.Y.

\section{COMPETING INTERESTS}

474 The authors declare no competing interests. 
Figures
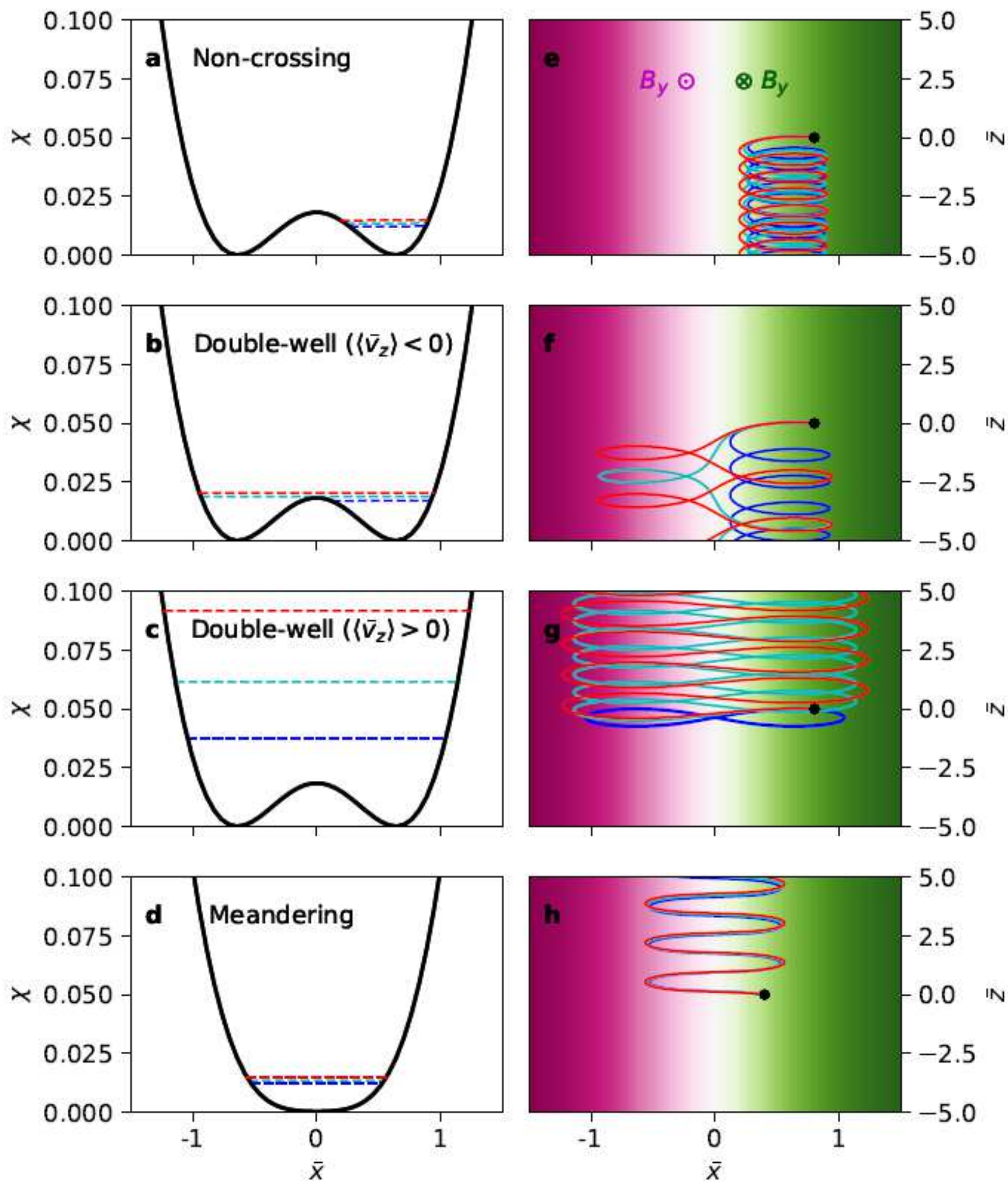

Figure 1

Four classes of particle orbits and their effective potentials. Please see manuscript .pdf for full caption. 

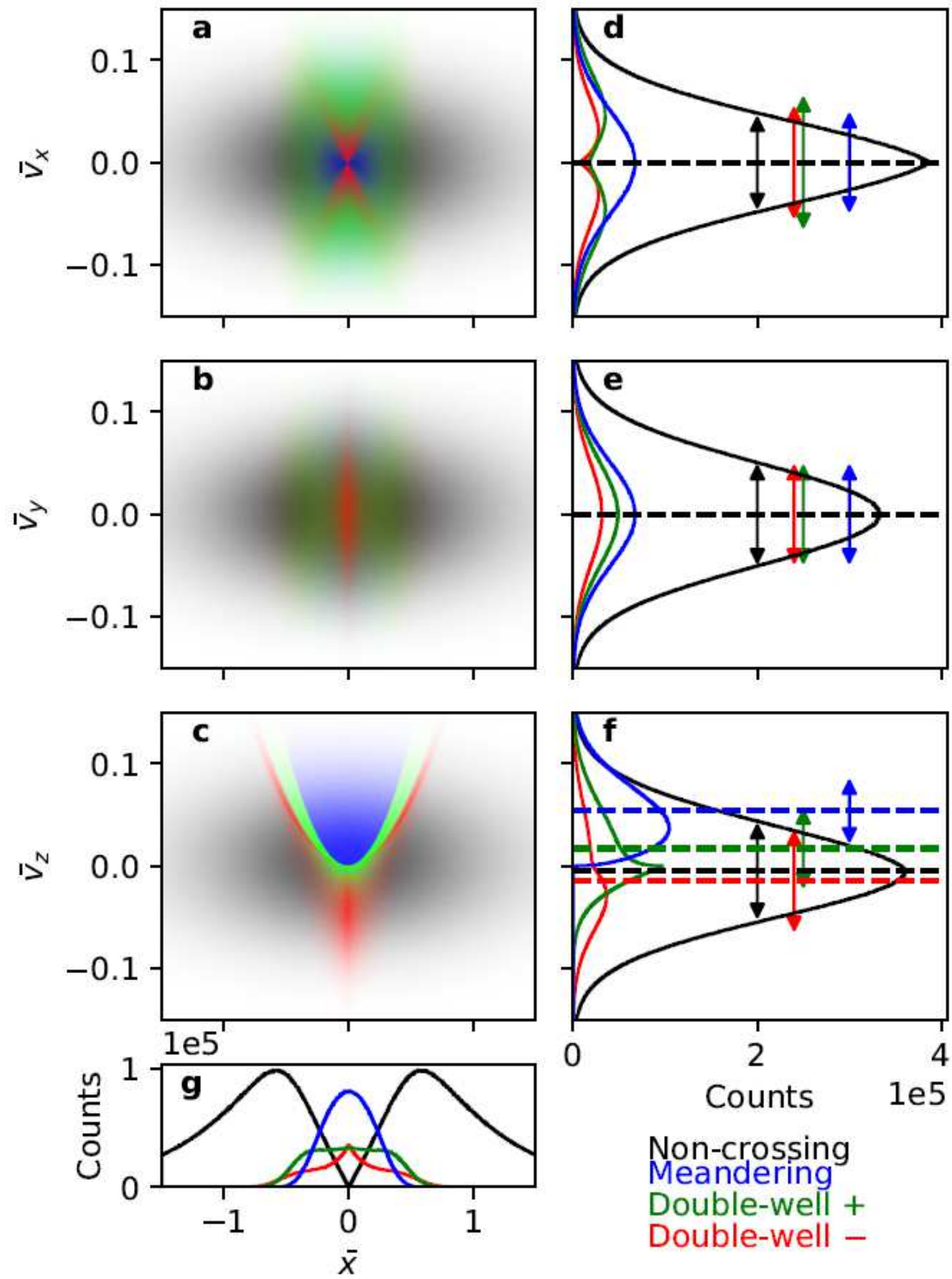

Non-crossing Meandering Double-well + Double-well -

Figure 2

Particle distribution in phase space, velocity space, and physical space. Please see manuscript .pdf for full caption. 

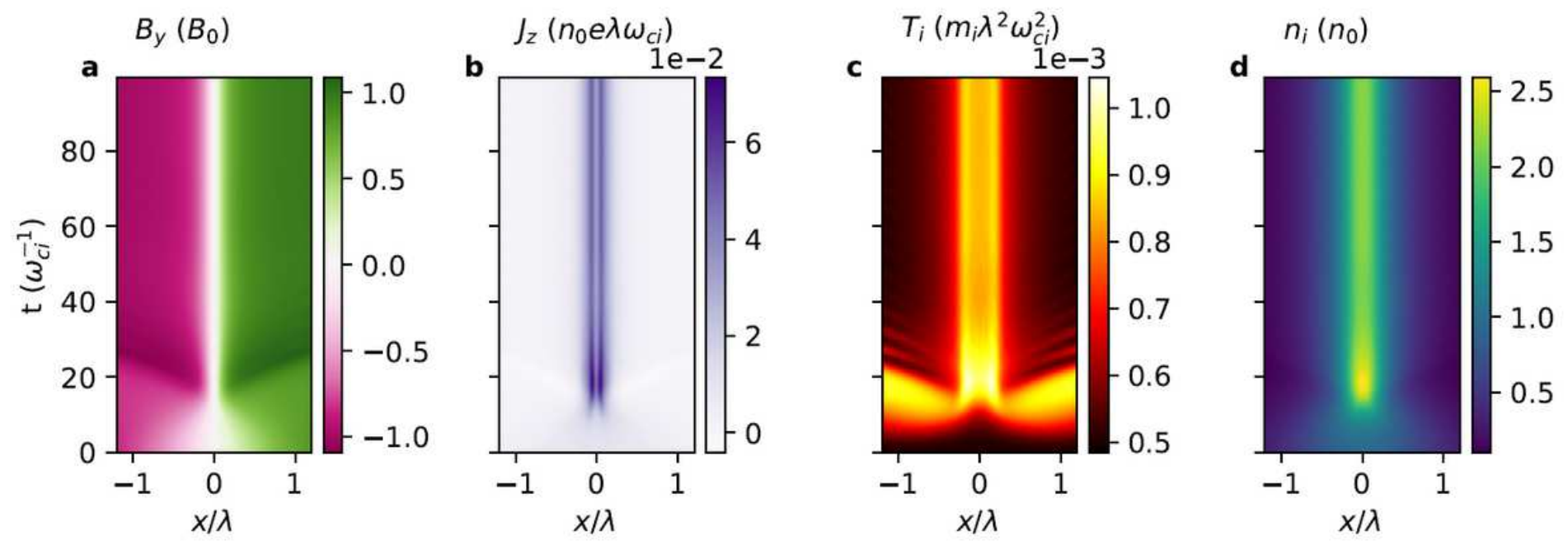

Figure 3

Streak plots of variables from the particle-in-cell simulation. Please see manuscript .pdf for full caption.
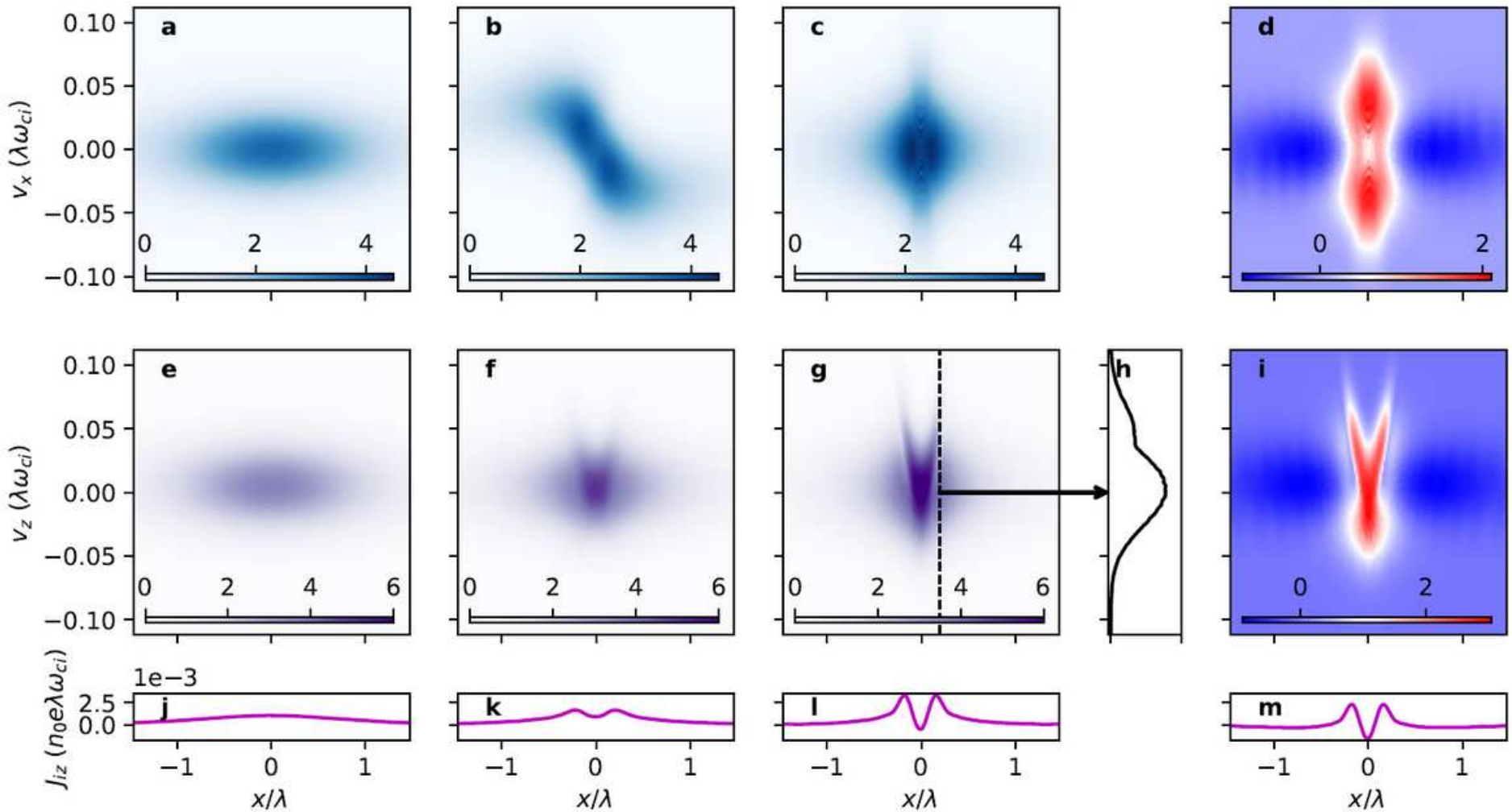

Figure 4

Time evolution of the ion distribution function from the particle-in-cell simulation. Please see manuscript .pdf for full caption. 

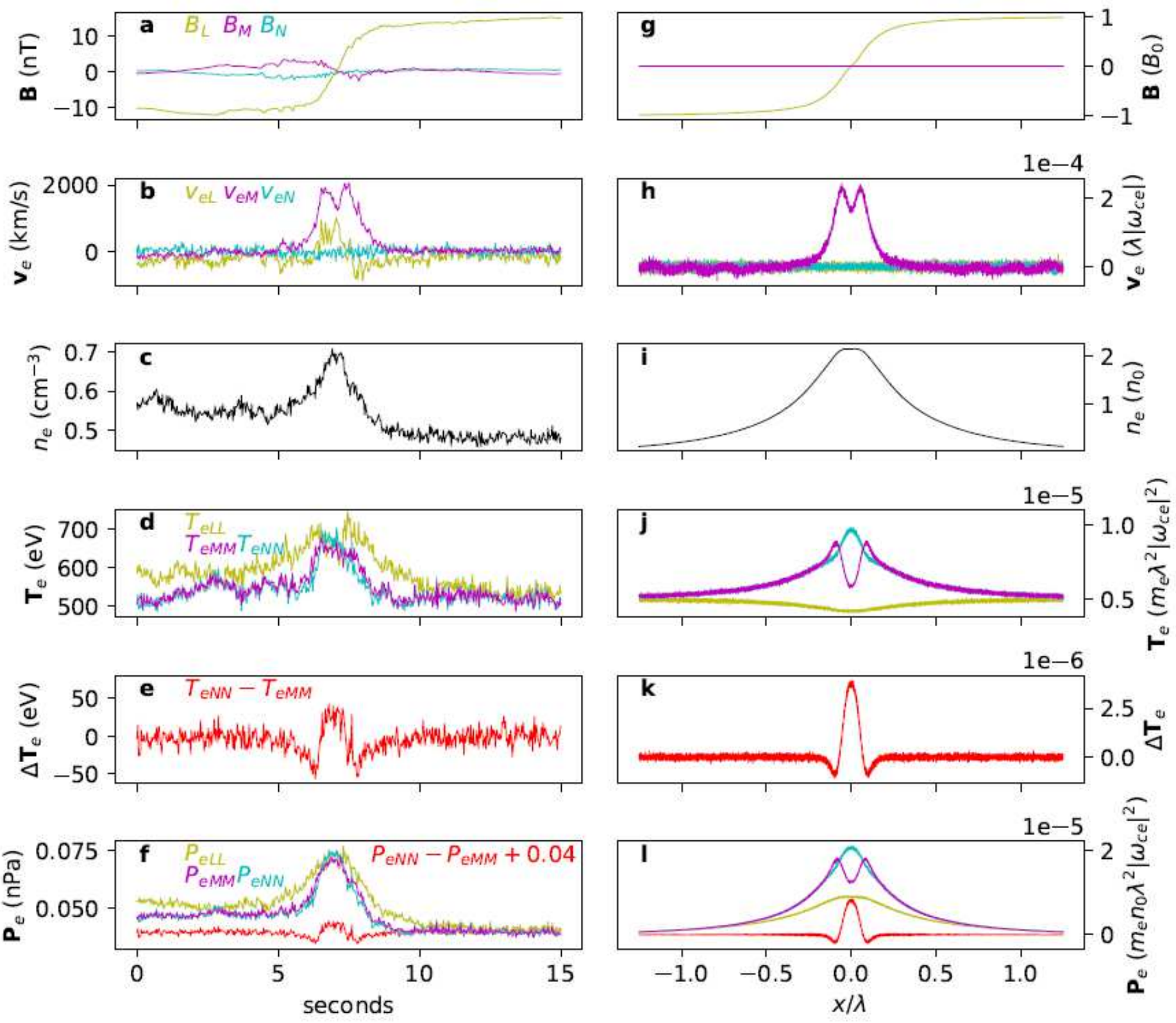

Figure 5

Comparison of a current sheet detected by MMS to that from the particle-in-cell simulation. Please see manuscript .pdf for full caption. 